\title{
Improving the Light Extraction Efficiency of GaN-Based Light-Emitting Diode
}

\author{
Lianjun Zhang, Zhongqi Fan, Gang Liu \\ Computer College Shandong University of Technology, Zibo, China \\ Email: zhlj0512@163.com
}

How to cite this paper: Zhang, L.J., Fan, Z.Q. and Liu, G. (2021) Improving the Light Extraction Efficiency of GaN-Based LightEmitting Diode. World Journal of Engineering and Technology, 9, 300-308. https://doi.org/10.4236/wjet.2021.92021

Received: March 31, 2021

Accepted: May 7, 2021

Published: May 10, 2021

Copyright (อ 2021 by author(s) and Scientific Research Publishing Inc. This work is licensed under the Creative Commons Attribution International License (CC BY 4.0).

http://creativecommons.org/licenses/by/4.0/ (c) (i) Open Access

\begin{abstract}
The light extraction efficiency caused by total internal reflection is low. Based on the analysis of the existing technology, a new design scheme is proposed in this paper to improve the light extraction efficiency. The air gap photonic crystal is embedded on the GaN-based patterned sapphire substrate, which can reduce line misalignment and improve light extraction efficiency. The internal structure of the GaN-based LED epitaxial layer is composed of an electron emission layer, a quantum well in the light-emitting recombination region, and an electron blocking layer. Experimental results show that this method significantly improves the extraction efficiency of LED light.
\end{abstract}

\section{Keywords}

Light Emitting Diodes, Light Extraction Efficiency, Photonic Crystals, Epitaxial Lateral Overgrowth

\section{Introduction}

Due to the small size and high efficiency of light-emitting diodes (LED), they are gradually becoming a substitute for traditional light sources. There is a band gap in GaN semiconductors, and the wavelength can be easily added by adding indium (transferred to longer wavelengths) or aluminum (transferred to longer wavelengths). To shorter wavelengths from the green to the blue region [1]. Nitride-based LEDs provide new opportunities for full-color LED displays and high-efficiency white light sources, which will replace incandescent light sources. GaN-based light-emitting diodes provide new opportunities for full-color lightemitting diode LED displays and white light sources.

The performance of light-emitting diodes is mainly determined by two advantages, namely the internal quantum efficiency (IQE) of the active area and the light extraction efficiency [2]. But the result of the low internal quantum ef- 
ficiency is affected by the high thread dislocation (TD) density of the GaN film grown on a heterogeneous substrate, TD is the metal material which forms an electron diffusion path that is injected into the active layer [3]. Due to the weaker positioning of the matrix, the light emission efficiency is more sensitive to the non-radioactive recombination center of TD when the emission wavelength is shortened. And the LED luminous efficiency decays under high current injection [4], the problem of (Efficiency droop) has not been effectively solved, which limits the development of high-power and high-brightness LEDs to a certain extent. In order to improve the performance of light-emitting diodes, it is necessary to design a suitable structure to improve the light extraction efficiency. The recent technology used to improve the light extraction efficiency, including the conventional surface crystal etching on the gas-phase GaN interface and the GaN substrate interface Technology, as well as the structure of the photonic crystal itself [5]. On this basis, this paper proposes a suitable LED emission model to improve luminous efficiency.

\section{Conventional Lattice Structure Surface Improves Light Extraction Efficiency}

Traditional methods to achieve light extraction efficiency include the improvement of the surface lattice structure of the material and the thin-film GaN substrate. The use of the surface lattice structure can significantly increase the light extraction efficiency. The key is to increase the probability of photon emission. Photonic crystals. Is a diffraction grating in a certain sense, as long as its grating constant is in the order of light wavelength or even sub-wave length. When light enters the photonic crystal material, it exhibits the wave properties of light. When the light wavelength is equal to the period of the medium, the grating structure occurs when acting, the light wave is modulated, so that the light falls in the emergent light cone and is coupled into the emergent light, thereby improving the extraction efficiency of the LED. In addition to the periodic photonic crystal structure, other uses of epitaxial lateral excessive growth (in n-GaN Layer) can also reduce TD. Embedded dielectric mask and patterned sapphire crystal substrate (PSS), these methods will improve the quality of the film, thereby increasing the internal quantum efficiency. In addition, for substrate materials with different refractive indices, such as $\mathrm{SiO}_{2}$ and have tilt the angular patterned sapphire PSS also effectively increases light scattering and reflection, and improves light extraction efficiency.

The extraction efficiency of the GaN-based LEDs remains low because of the total reflection that occurs at the air-GaN interface. As a result, the light is trapped inside the semiconductor, and will eventually be absorbed by defects and recombination centers in the substrate or the active region [4]. Only lights within the extract cone can exit from the interface of the semiconductor and air.

Where are the refractive index of the air and GaN $(2.5$ at $0.44 \mu \mathrm{m})$, respectively. The effect of liquid-phase-deposited (LPD) ZnO rods on the LED surface was also investigated [6]. The LPD method has near room temperature deposi- 
tion, atmospheric growth and low cost. The key idea here was to increase the range of the escape angle. The escape angle of was spread from 43.9 to 53.2 for the light emission from the $\mathrm{GaN}$ and get out from the $\mathrm{ZnO}$ side walls.

\section{Surface Photonic Crystals Used to Improve the Light Extraction}

Recently, photonic crystals structures are used on the surface of the III-nitride LEDs to improve the light extraction efficiency. Photonic crystal can be used to inhibit the spontaneous emission spectrum [7], or used as a diffraction grating to increase the internal extraction efficiency. They can also enhance the internal quantum efficiency by means of the Purcell effect.

Figure 1, can seed that the deep etched and shallow etched photonic crystal both enhanced the light extraction efficiency. Although the deep etched photonic crystal had low internal quantum efficiency, the light output was still higher than the shallow etched one and the planar surface LED.

\subsection{Surface Photonic Crystals Used as Diffraction Grating}

When using the surface photonic crystal of the diffraction grating to emit photons, it allows spontaneous emission guided mode, but this mode has the properties of coupled diffraction [8]. The extra Brock momentum generated by the periodic mode makes the light and the leaky state form a coupling. It can not only improve the efficiency of light extraction, but also control the radiation pattern. In this method, the lattice constant of the diffraction grating is larger than the band gap. In this approach, the lattice constant of a diffraction grating is larger than in the band-gap approach, since the photonic crystal operates near a high Bragg order rather than at the first Bragg order.

$$
k_{\| m}=(2 \pi / \lambda) n_{\mathrm{GaN}} \sin \left(\theta_{m}\right)=\sqrt{\left((2 \pi / \lambda) n_{\mathrm{GaN}}\right)^{2}-(m \pi / L)^{2}}
$$

where $m$ is the mode number and $L$ is thickness of the GaN. With the presence of $\mathrm{GaN}$, the wave vector is now coupled to other harmonic modes by the reciprocal lattice vectors $G$.

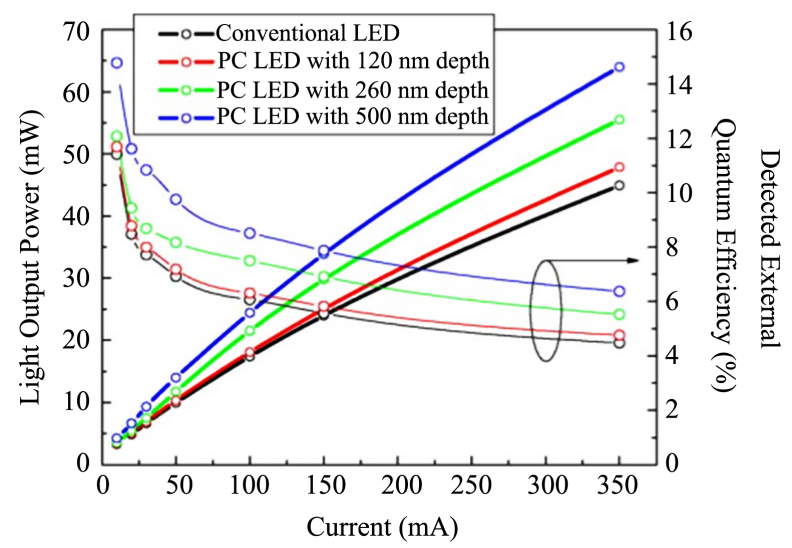

Figure 1. Light output power and current characteristics. 


$$
\left|k_{\| m}+G\right|<2 \pi / \lambda
$$

In order to diffract the guided light into the air, the necessary conditions need to be met. The diffraction conditions strongly depend on the wavelength of light and the lattice constant. Due to the multi-mode nature of GaN, the coupling of the leakage mode and the photonic crystal should be considered. The higher-order modes are usually higher than the lower-order modes. Mode diffraction is better because they have better overlap with photonic crystals. Another way to ensure better coupling is to embed the photonic crystal structure in the middle of the LED device [9]. Use $700 \mathrm{~nm}$ thick GaN and a lattice constant of $250 \mathrm{~nm}$ The specification photonic crystal experiment shows that the light extraction efficiency is improved. Compared with the rough surface thin film LED, the extraction efficiency of the photonic crystal LED is as high as (65\% - 78\%) (Figure 2).

\subsection{Patterned Sapphire Substrate to Improve GaN Film Quality and Scatter Reflected Light}

The most commonly seen patterned sapphire substrate has periodic pyramid arrays with 3 facets. This has numerically studied the effect of pyramid angles on the light extraction efficiency (LEE) for 4-facet pyramid structures [10]. The result suggested $30^{\circ}$ slanted angle could be the best angle in enhancing light extraction efficiency (Figure 3 ).

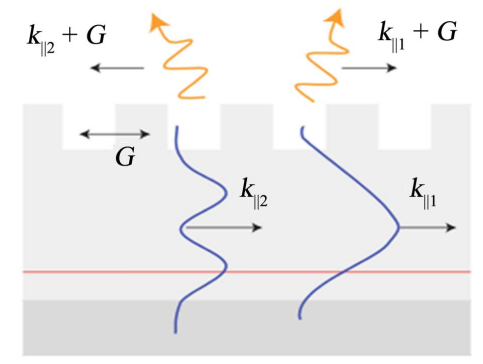

Figure 2. Guided modes in the LED structure with photonic crystals.

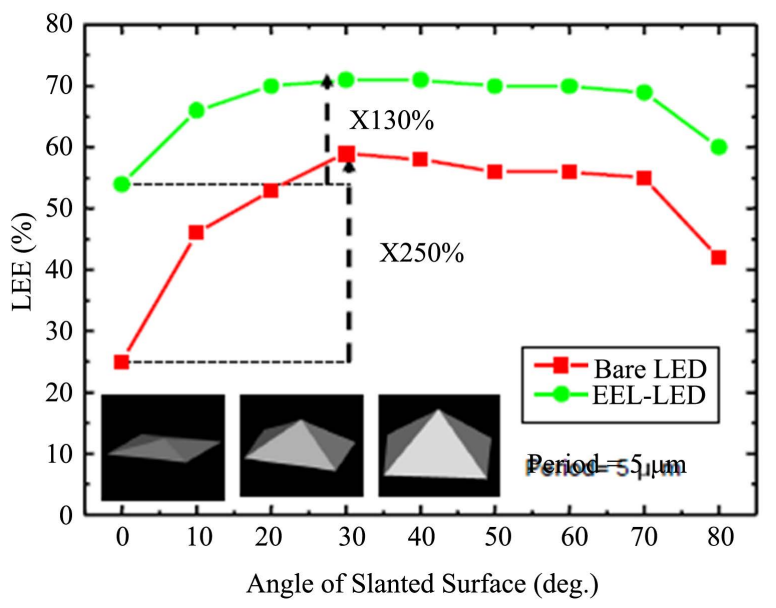

Figure 3. The LEE angle of the slanted surface in an inversed pyramid structure. 
Experimental studies have shown that adjusting the tilt angle to $31.6^{\circ}$ will produce better performance [11]. The patterned sapphire substrate with an angle of $31.6^{\circ}$ produces $66 \%$ IQE and $20 \mathrm{~mW}$ light output at $20 \mathrm{~mA}$ current. The reason is the increase in light extraction efficiency. Especially the difference in crystal quality is determined by the lateral growth area. It is determined by the c-plane area, a small oblique angle will have a small c-plane area. The dislocation density is characterized by X-ray diffraction and etch pit density. An experiment is made. The tapered PSS is shown in Figure 4 to improve the extraction efficiency [12]. The lattice constant is designed to be $3 \mu \mathrm{m}$, and the cone diameter is 2.5 $\mu \mathrm{m}$. The etching depth and sidewall angle are about $700 \mathrm{~nm}$ and $29^{\circ}$. Compared with conventional LED, the light output power is higher. There is a $35 \%$ enhancement due to the light reflecting from the sapphire substrate to the top direction, reducing thread dislocations.

It is also possible to use deposited silicon dioxide spheres on the sapphire substrate PSS to realize the MOCVD method of heteroepitaxial growth of thin films at a lower manufacturing cost, and to reduce the dislocation of the GaN film [13]. The diameter of the silicon spheres ranges from $300-550 \mathrm{~nm}$. Change, the surface coverage is $15 \%-70 \%$. The results show that a higher coverage and larger sphere size will give better results. In addition to the cone shape and colloidal sphere mode, the PSS of the irregular nanoarray is in It is also effective in improving the external quantum efficiency [14]. There are many accumulation faults in the gaps between the $\mathrm{SiO}_{2}$ nanorods, and visible thread dislocations are rarely observed in the vicinity. The formation of dislocations on the sapphire surface is further reduced.

Previously analyzed many structures that can improve light extraction efficiency, including surface and embedded photonic crystals, embedded dielectric film shielding and patterned sapphire substrates. However, the low-order mode light guide in GaN weakly interacts with photonic crystals, making photonic crystals cannot effectively extract most of the light. In order to extract all the light guide modes, the use of embedded air gap photonic crystal is a better choice. Due to a large number of thread dislocations, the quality of the $\mathrm{GaN}$ film is poor,

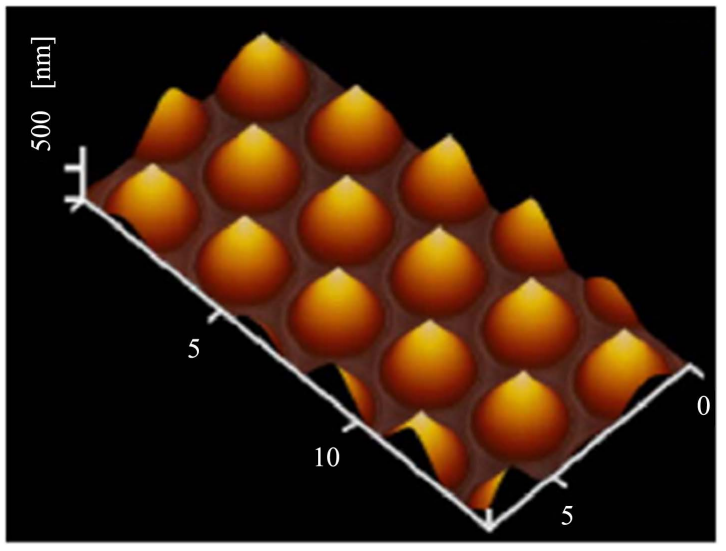

Figure 4. AFM images of $31.6^{\circ}$ tilt view of sapphire surface with PSS. 
and the internal quantum efficiency will be reduced. The patterned sapphire substrate reduces the impact. In order to achieve good external quantum efficiency, it is necessary to use the air gap photonic crystal to obtain high light extraction efficiency, while improving the quality of GaN film to achieve high internal quantum efficiency.

The coalescence front of the GaN seed crystal provides a strain relief layer in which stacking defects may occur, and most of them are found to be located above the voids or smaller GaN seed crystals. If the growth rate is too slow to become a $\mathrm{GaN}$ seed crystal, dislocations can be prevented by forming voids. The remaining $\mathrm{SiO}_{2}$ between the nanorods can prevent the growth of $\mathrm{GaN}$ and further reduce the formation of dislocations from the sapphire surface.

There have been many structures that can enhance light extraction efficiency, including surface and embedded PhCs, embedded dielectric ELO masks and patterned sapphire substrates. The surface PhCs can been low order mode guided light in GaN interacts weakly with surface PhCs and this large portion of light cannot be extracted efficiently. In order to extract all guided light modes, the embedded PhCs are better candidates and air-gap $\mathrm{PhC}$ might provide the highest efficiency because the refractive index to air which has the highest contrast to GaN.

\section{An New Structure Embedded Design in Air Gap Photonic Crystal}

With a large amount of threading dislocations, the GaN film quality is poor and the intrinsic quantum efficiency will be lower. In order to achieve good external quantum efficiency, we need to use air-gap $\mathrm{PhCs}$ to obtain high light extraction efficiency and simultaneously improve GaN film quality to achieve high internal quantum efficiency.

In order to improve the light extraction efficiency, embedded air gap photonic crystals, embedded dielectric film shielding and patterned sapphire substrates are used. For photonic crystals, the low-order mode light guide in GaN weakly interacts with the surface photonic crystal, and the surface Photonic crystals cannot effectively extract most of this light. In order to extract all light guide modes, it is better to embed photonic crystals with void photonic crystals, which can provide the highest efficiency, because the refractive index of air is 1 , such cones and GaN The difference in refractive index of the interface is the largest. Since the air gap photonic crystal does not have the function of epitaxial lateral growth, if an embedded dielectric mask is used, it can be embedded after the re-growth after the air gap is formed.

Compared with other designs, the device structure obtained by this design has very few dislocations. At the same time, the advantages of the air gap photonic crystal are used to extract most of the guidance modes. The experimental results show that the extraction efficiency of the photonic crystal LED is as high as (75\% - 83\%). In this structure, both the internal quantum efficiency and light extraction 


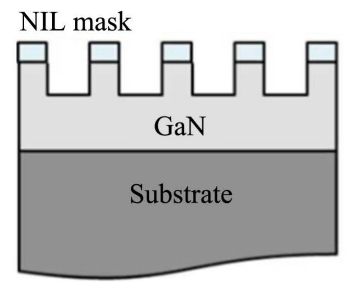

(a)

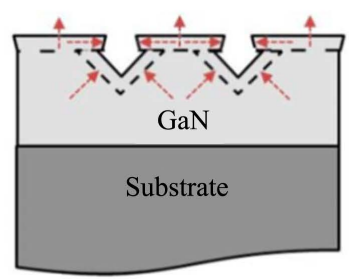

(b)

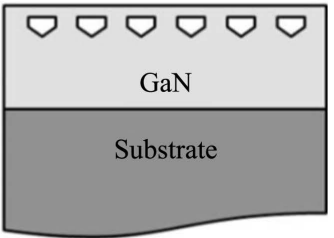

(c)

Figure 5. (a) PhC was patterned by NIL and transferred to GaN; (b) Semipolar facets formed during regrowth by MOCVD; (c) Air-gap PhCs were embedded after the overgrowth of GaN.

efficiency will be improved, and the external quantum efficiency will increase dramatically.

The thread dislocations continue to be photonic in the air gap On-crystal growth. In the buffer GaN layer, fewer dislocations can be formed. According to the above theoretical analysis, this paper designs a new type of sapphire substrate PSS to form an embedded air gap photonic crystal LED. First, by hot press lithography (NIL) the photonic crystal mode GaN template obtained on the sapphire substrate (Figure 5(a)). Then use MOCVD [11] method to overgrow GaN (Figure 5(b)), under the optimized growth conditions in (0001). After the gap generated by the growth rate of the plane, the layer structure is overgrown (Figure 5(c)).

The resulting device structure will have few dislocations compared to work, and at the same time use the advantage of the air gap PhC to extract most of the guided modes [15] [16]. The structure proposed in this paper can improve these situations, so that both the internal quantum efficiency and light extraction efficiency will be improved, and the external quantum efficiency is expected to be significantly improved.

\section{Conclusions}

Both surface and embedded $\mathrm{PhC}$ can be used as diffraction gratings, and can effectively extract guided modes in GaN. The embedded dielectric mask can improve the quality of the GaN film by epitaxial lateral growth, and also scatter the reflected light back to the top surface to achieve better light extraction. The size of the dielectric mask can be micron size or nanometer size, and the total mask area available is very important. The patterned sapphire substrate can also reduce threading dislocation density and provide better film quality, and effectively reflect light back to the top surface.

These technologies can improve the internal quantum efficiency by improving the quality of the GaN film, and most importantly, the use of suitable structures, the use of surface textures, patterned substrates, and surface/embedded photonic crystals to improve light extraction efficiency. With optimized device structure design and manufacturing technology, GaN blue LEDs paved the way for lowcost, high-performance solutions to meet the growing demand for full-color displays and energy-saving lighting in the future. 


\section{Conflicts of Interest}

The authors declare no conflicts of interest regarding the publication of this paper.

\section{References}

[1] Chuang, S.L. (2009) Physics of Photonic Devices. 2nd Edition, John Wiley \& Sons, Inc., Hoboken.

[2] Gao, H., Yan, F., Zhang, Y., Li, J., Zeng, Y. and Wang, G. (2008) Enhancement of the Light Output Power of InGaN/GaN Light-Emitting Diodes Grown on Pyramidal Patterned Sapphire Substrates in the Micro-Nanoscale. Journal of Applied Physics, 103, Article ID: 014314. https://doi.org/10.1063/1.2830981

[3] Zang, K.Y. (2010) Nanoepitaxy to Improve the Efficiency of InGaN Light-Emitting Diodes. Applied Physics Letter, 92, 43126-43131.

[4] Wierer, J., David, A. and Megens, M.M. (2009) III-Nitride Photonic-Crystal LightEmitting Diodes with High Extraction Efficiency. Nature Photonics, 3, 3214-3220. https://doi.org/10.1038/nphoton.2009.21

[5] Piprek, J. (2010) Efficiency Droop in Nitride-Based Light-Emitting Diodes. Physica Status Solidi $(A), 207,2217-2225$. https://doi.org/10.1002/pssa.201026149

[6] Lee, M.K., Ho, C.-L. and Chen, P.-C. (2008) Light Extraction Efficiency Enhancement of GaN blue LED by Liquid Phase Deposited ZnO Rods. IEEE Photonics Technology Letters, 20, 252-254. https://doi.org/10.1109/LPT.2007.913652

[7] Matioli, E., Fleury, B., Rangel, E., Melo, T., Hu, E., Speck, J., et al. (2010) High Extraction Efficiency GaN-Based Photonic Crystal Light-Emitting Diodes. Applied Physics Express, 3, Article ID: 032103. https://doi.org/10.1143/APEX.3.032103

[8] Kramers, M.R., Shchekin, O.B., Mueller-Mach, R., Mueller, G.O., Zhou, L., Harbers, G., et al. (2009) Status and Future of High-Power Light-Emitting Diodes for Solid-State Lighting. Journal of Display Technology, 3, 160-175. https://doi.org/10.1016/j.sse.2008.05.005

[9] Lee, H.C., Park, J.B., Bae, J.W., Thuy, P.T.T., Yoo, M.C. and Yeom, G.Y. (2008) Effect of the Surface Texturing Shapes Fabricated Using Dry Etching on the Extraction Efficiency of Vertical Light-Emitting Diodes. Solid-State Electronics, 52, 1193 1196. https://doi.org/10.1016/j.sse.2008.05.005

[10] Huang, S., Ye, Z., Lu, J., Su, Y., Chen, C. and He, G. (2013) Position Dependence of Extraction Efficiency in Organic Light-Emitting Diodes with Photonic Crystal Structure. Chinese Optics Letters, 11, Article ID: 062302.

[11] Cheng, J.H., Wu, Y.C.S., Liao, W.-C. and Lin, B.-W. (2014) Improved Crystal Quality and Performance of GaN-Based Light-Emitting Diodes by Decreasing the Slanted Angle of Patterned Sapphire. Applied Physics Letters, 96, Article ID: 051109. https://doi.org/10.1063/1.3304004

[12] Huang, H.W., Huang, J.K., Lin, C.H., Lee, K.Y., Hsu, H.W. Yu, C.C. and Kuo, H.C. (2010) Efficiency Improvement of GaN-Based LEDs with a SiO2 Nanorod Array and a Patterned Sapphire Substrate. IEEE Electron Device Letters, 31, 582-584. https://doi.org/10.1109/LED.2010.2045472

[13] Ueda, K., Tsuchida, Y., Hagura, N., Iskandar, F., Okuyama, K. and Endo, Y. (2010) High Performance of GaN Thin Films Grown on Sapphire Substrates Coated with a Silica-Submicron-Sphere Monolayer Film. Applied Physics Letters, 92, Article ID: 101101. https://doi.org/10.1063/1.2891067 
[14] Chiu, C.H., Yen, H.H., Chao, C.L., Li, Z.Y., Yu, P.C., Kuo, H.C., et al. (2011) Nanoscale Epitaxial Lateral Overgrowth of GaN-Based Light-Emitting Diodes on a $\mathrm{SiO}_{2}$ Nanorod-Array Patterned Sapphire Template. Applied Physics Letters, 93, Article ID: 081108. https://doi.org/10.1063/1.2969062

[15] Zhang, Y.Y., Fan, G.H., Yin, Y.A. and Yao, G.R. (2012) Performance Enhancement of Blue Light-Emitting Diodes without an Electron-Blocking Layer by Using Special Designed P-Type Doped InGaN Barriers. Optics Express, 20, A133-A140.

https://doi.org/10.1364/OE.20.00A133

[16] Zhao, Z., Li, G., Yu, F., Yang, H., Chen, X. and Lu, W. (2018) Sub-Wavelength Grating Enhanced Ultra-Narrow Graphene Perfect Absorber. Plasmonics, 13, $2267-$ 2272. https://doi.org/10.1007/s11468-018-0748-9 